\title{
Experience with combination of nimotuzumab and intensity-modulated radiotherapy in patients with locoregionally advanced nasopharyngeal carcinoma
}

This article was published in the following Dove Press journal:

OncoTargets and Therapy

18 November 2015

Number of times this article has been viewed

\section{Rui-ping Zhai \\ Hong-mei Ying \\ Fang-fang Kong \\ Cheng-run Du \\ Shuang Huang \\ Jun-jun Zhou \\ Chao-su Hu}

Department of Radiation Oncology, Fudan University Shanghai Cancer Center, Shanghai, People's Republic of China
Correspondence: Hong-mei Ying Department of Radiation Oncology, Fudan University Shanghai Cancer Center, 270 Dong'an Road, Shanghai 200032, People's Republic of China Tel +862164175590 Fax +862164175590 Email yinghongmei2013@163.com
Aim: To evaluate the efficacy and safety of using nimotuzumab in combination with intensitymodulated radiotherapy (IMRT) in the primary treatment of locoregionally advanced nasopharyngeal carcinoma.

Methods: Between December 2009 and December 2013, 38 newly diagnosed patients with stage III-IV nasopharyngeal carcinoma were treated with IMRT and nimotuzumab concomitantly. The distribution of disease was stage III in 20 (52.6\%), stage IV A in 9 (23.7\%), and stage IV $\mathrm{B}$ in $9(23.7 \%)$. All the patients received at least two cycles of cisplatin-based neoadjuvant chemotherapy followed by nimotuzumab $200 \mathrm{mg} /$ week concurrently with IMRT. Acute and late radiation-related toxicities were graded according to the Acute and Late Radiation Morbidity Scoring Criteria of Radiation Therapy Oncology Group.

Results: With a median follow-up of 39.7 months (range, 13.3-66.5 months), the estimated 3-year local recurrence-free survival, regional recurrence-free survival, distant metastasisfree survival, progression failure-free survival, and overall survival rates were $92.8 \%, 92.9 \%$, $89.5 \%, 78.7 \%$, and $87.5 \%$, respectively. The median cycle for nimotuzumab addition was 6 weeks. Grade 3 radiation-induced mucositis accounted for $36.8 \%$ of treated people. No skin rash and infusion reaction were observed, distinctly from what is reported in cetuximab-treated patients.

Conclusion: Nimotuzumab plus IMRT showed promising outcomes in terms of locoregional control and survival, without increasing the incidence of radiation-related toxicities for patients.

Keywords: nasopharyngeal carcinoma, intensity-modulated radiotherapy, nimotuzumab

\section{Introduction}

Concomitant chemoradiotherapy (CRT) is the standard of care for locoregionally advanced nasopharyngeal carcinoma (LA NPC), with established benefit in overall survival (OS). ${ }^{1}$ But the treatment-related toxicities are also considerable. Grade 3-4 radiation-related mucositis was reported to range from $45.4 \%$ to $62 \%$ in several series. ${ }^{2-4}$ Also the benefit of CRT is still uncertain in the intensity-modulated radiotherapy (IMRT) era, since the locoregional tumor control of nasopharyngeal carcinoma (NPC) has been significantly improved with the use of IMRT. ${ }^{5}$ Therefore, it is vital for clinicians to explore new agents that could enhance the efficacy of IMRT, while inducing minimal toxicities when concomitantly was used with IMRT.

It has shown that overexpression of EGFR is a feature of a variety of human malignancies including NPC, and high EGFR expression was associated with poor prognosis..$^{6-9}$ Cetuximab, the most commonly used anti-EGFR antibody, combined 
with radiotherapy (RT), has been shown to improve survival in patients with locoregionally advanced head and neck squamous cell carcinoma (LA HNSCC). ${ }^{10}$ In NPC, the schedule of concurrent cetuximab-cisplatin-IMRT has achieved promising preliminary results. However, the relatively high rate of mucositis and acne-like skin rash limited its clinical application. ${ }^{11-13}$

Nimotuzumab is a humanized monoclonal antibody that is obtained by replacing a murine complementary-determining regions with a human framework. In the preclinical studies, nimotuzumab has demonstrated remarkably antiproliferative, proapoptotic, and antiangiogenic activities. ${ }^{14}$ Nimotuzumab combined with irradiation or chemoradiation has shown clinical efficacy in improving locoregional control and OS in patients with LA HNSCC. ${ }^{15,16}$ However, there is little research focusing on nimotuzumab and IMRT for LA NPC. Therefore, the purpose of this study is to evaluate the safety and efficacy of nimotuzumab plus IMRT in patients with LA NPC.

\section{Patients and methods}

\section{Patients and pretreatment}

From December 2009 to December 2013, 38 patients (median 46 years; range 18-65 years) with stage III or stage IV NPC (histologically proven World Health Organization type II or III) were treated with nimotuzumab plus definitive IMRT in Fudan University Shanghai Cancer Center. The pretreatment workup included a complete historical physical examination, magnetic resonance imaging (MRI) scans of the head and neck, chest radiography, abdominal ultrasonography, emission computed tomography, and blood samples for hematological, biochemical, and serological estimations. All patients were staged according to the Union for International Cancer Control 2010 staging system. Clinical characteristics of the patients are listed in Table 1. The authors state that all procedures performed in the study were in accordance with the ethical standards of our Fudan University Shanghai Cancer Center research committee or have followed the principles outlined in the Declaration of Helsinki for all human or animal experimental investigations. For investigations involving human subjects, written informed consent was obtained from all individual participants included in this study.

\section{Intensity-modulated radiotherapy}

All patients were treated with definitive IMRT, following a consistent dose and volume prescription policy. The gross tumor volume (GTV) was defined in accordance with the International Commission on Radiation Units and
Table I The clinical characteristics of 38 patients enrolled in the study

\begin{tabular}{|c|c|}
\hline $\begin{array}{l}\text { Patient } \\
\text { characteristics }\end{array}$ & $\begin{array}{l}\text { Number of patients } n(\%) \\
(n=38)\end{array}$ \\
\hline \multicolumn{2}{|l|}{$\overline{S e x}$} \\
\hline Male & $25(65.8)$ \\
\hline Female & I3 (34.2) \\
\hline \multicolumn{2}{|l|}{ Age, years } \\
\hline Median & 46 \\
\hline$<46$ & I 8 (47.4) \\
\hline$\geq 47$ & $20(52.6)$ \\
\hline \multicolumn{2}{|c|}{ WHO histologic type } \\
\hline II & $6(15.8)$ \\
\hline III & $32(84.2)$ \\
\hline \multicolumn{2}{|l|}{ T stage } \\
\hline TI & $6(15.8)$ \\
\hline $\mathrm{T} 2$ & $8(2 I . I)$ \\
\hline T3 & $13(34.2)$ \\
\hline $\mathrm{T} 4$ & II (28.9) \\
\hline \multicolumn{2}{|l|}{$\mathrm{N}$ stage } \\
\hline No & I (2.6) \\
\hline NI & $7(18.4)$ \\
\hline N2 & $21(55.3)$ \\
\hline N3 & $9(23.7)$ \\
\hline \multicolumn{2}{|l|}{ UICC stage } \\
\hline III & $20(52.6)$ \\
\hline IV A & $9(23.7)$ \\
\hline IV B & $9(23.7)$ \\
\hline \multicolumn{2}{|l|}{ Nimotuzumab } \\
\hline$<6$ cycles & $8(2 I .1)$ \\
\hline$\geq 2$ cycles & $30(78.9)$ \\
\hline
\end{tabular}

Abbreviations: UICC, Union for International Cancer Control; WHO, World Health Organization; T, tumor; N, lymph node.

Measurements Reports 50 and 62, including all gross primary tumors (GTV-P) and involved lymph nodes (GTV-LN) determined from clinical and imaging examinations. An additional $5 \mathrm{~mm}$ margin around the GTV-P and GTV-LN was required in all directions to define planning target volume (PTV-G and PTV-LN), respectively. The clinical target volume (CTV) was defined as the GTV plus $0.5-1 \mathrm{~cm}$ margin to encompass any subclinical disease, together with the areas of lymph nodes drainage. The CTV for high-risk (CTV-H) should include the entire nasopharyngeal cavity, the parapharyngeal space, pterygoid fossa, retropharyngeal lymph nodal regions, the posterior of the nasal cavity and the maxillary sinus, the anterior half of the clivus (when invaded, the whole clivus was covered), the skull base, the inferior sphenoid sinus and any high-risk nodal regions, including the regional lymphatics of level II, III, and V A and the station of the positive lymph nodes. The neck area of lymph node drainage was outlined according to the Recommendation by the Radiation Therapy Oncology Group (RTOG) consensus guidelines. ${ }^{17,18}$ The CTV for low-risk (CTV-L) was recognized as the next station of the positive lymph nodes without any evidence of 
metastatic lymph nodes (generally level IV and level V B). Level I B was irradiated selectively only in patients who had extensive nodal involvement on the ipsilateral II A region or extracapsular extension of the II A lymph nodes. The PTV-C would cover CTV with a $3 \mathrm{~mm}$ margin in all directions. When the CTV is near the critical organs at risk (OAR), such as the spinal cord and brain stem, the margin would be contracted to be as small as $1 \mathrm{~mm}$.

The OAR included spinal cord, brain stem, temporal lobes, optic nerves, chiasm, parotid glands, eyeballs, lens, temporomandibular joints, oral mucosa, and larynx. A $5 \mathrm{~mm}$ margin was added to the spinal cord and the brain stem to form the planning OAR volume during computerized optimization.

The treatment was performed with simultaneous integrated boost technique, using $6 \mathrm{MV}$ photons. The prescribed radiation dose was 66 or 70.4 Gy to PTV-G (66 Gy for T1 and T2 stage and 70.4 Gy for T3 and T4 stage), 66 Gy to PTV-LN, 60 Gy to PTV-CTV-H, and 54 Gy to PTV-CTV-L, delivered in 30 or 32 fractions. Computerized optimization was used with fusion of MRI and treatment planning computed tomography images, to accurately delineate the target. Radiation was delivered once daily, five fractions per week, over 6 weeks. The volume of PTV encompassed by less than $95 \%$ of the prescription dose should not exceed $1 \%$. More than $110 \%$ of the prescription dose was not allowed in or out of PTV. The dose to OAR was limited on the basis of the RTOG 0225 protocol.

\section{Chemotherapy}

Nimotuzumab was administered concomitantly with IMRT at a dose of $200 \mathrm{mg}$ weekly, which was diluted in $250 \mathrm{~mL}$ saline to obtain a $200 \mathrm{mg}$ suspension and intravenously infused over 1 hour. Seventy-eight percent of the patients received at least six cycles of nimotuzumab during IMRT. All the patients were given two to three cycles of platinumbased neoadjuvant chemotherapy and $64.1 \%$ of the patients had one to two cycles of adjuvant chemotherapy. The most common regimens of neoadjuvant and adjuvant chemotherapy included TPF (docetaxel $60 \mathrm{mg} / \mathrm{m}^{2} /$ day on day 1 , cisplatin $25 \mathrm{mg} / \mathrm{m}^{2} /$ day on days $1-3$, and 5-fluorouracil $500 \mathrm{mg} / \mathrm{m}^{2} /$ day on days $1-3$ ), TP (docetaxel $60 \mathrm{mg} / \mathrm{m}^{2} /$ day on day 1 , cisplatin $25 \mathrm{mg} / \mathrm{m}^{2} /$ day on days $1-3$ ), and GP regimen (gemcitabine $1,000 \mathrm{mg} / \mathrm{m}^{2} /$ day on days 1 and 8 , cisplatin $25 \mathrm{mg} / \mathrm{m}^{2} /$ day on days $1-3$ ).

\section{Patient evaluation}

The assessment of tumor response was performed twice after the completion of induction chemotherapy and 3 months after radiation, which was based on MRI according to Response Evaluation Criteria for Solid Tumors criteria. Systemic chemotherapy adverse effects were scored by using the National Cancer Institute Common Toxicity Criteria (NCI CTCAE, version 3.0), whereas RT-induced toxicities were scored according to the Acute and Late Radiation Morbidity Scoring Criteria of RTOG.

All the subjects underwent weekly examinations for treatment response and toxicities during radiation therapy. Patients were followed-up every 3 months in the first 2 years; every 6 months from the third to the fifth year, and then annually. Each follow-up included careful examination of the nasopharynx and neck nodes by an experienced doctor, MRI scan of the nasopharynx, chest computed tomography radiograph, and ultrasound of abdomen were performed 3 months after the completion of RT and every 6-12 months thereafter. Additional examinations were performed when it is indicated to evaluate local relapse or distant metastasis.

\section{Statistical analysis}

All the statistical analyses were performed with the Statistical Package for Social Sciences (IBM Corporation, Armonk, NY, USA) software. The main purpose of this investigation was to evaluate the efficacy and adverse events of nimotuzumab combined with IMRT in LA NPC. The estimated OS, progression failure-free survival (PFS), local recurrence-free survival (LRFS), regional recurrence-free survival (RRFS), and distant metastasis-free survival (DMFS) were calculated by the Kaplan-Meier method. Univariate analysis was performed with log-rank test. Significant factors with a $P$-value $<0.05$ were included in a multifactor Cox model to determine the independent prognostic factors. The duration of survival was defined as the commencement date of chemotherapy to the event of interest occurring or the last follow-up for the patients alive.

\section{Results \\ Response of disease}

The overall response rates were 100\% (complete remission [CR] 92.1\%) and 100\% (CR 92.1\%), respectively, for lesions of nasopharynx and cervical lymph nodes 3 months after the completion of radiation. The residual tumor gradually disappeared or became less obvious at the following 6-9 months.

\section{Local control and survival rates}

The median follow-up was 39.7 months (range, 13.3-66.5 months). The estimated 3-year LRFS, RRFS, DMFS, PFS, and OS rates were $92.8 \%, 92.9 \%, 89.5 \%, 76.3 \%$, and 


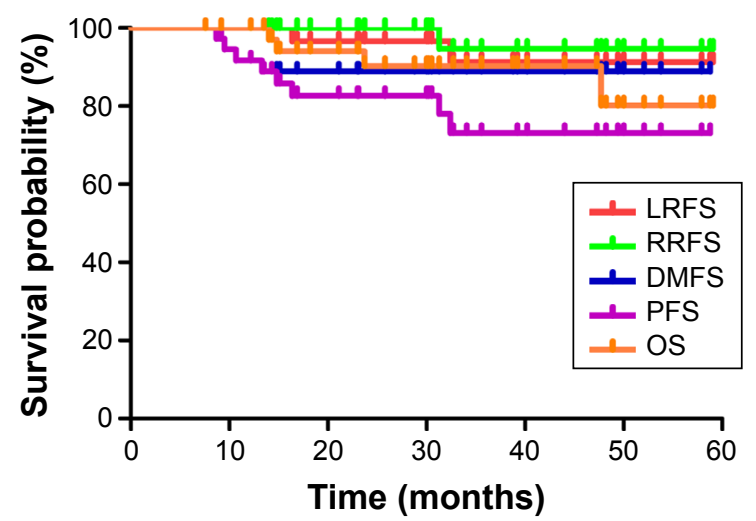

Figure I Kaplan-Meier curves showing LRFS, RRFS, DMFS, PFS, and OS of patients.

Abbreviations: DMFS, distant metastasis-free survival; LRFS, local recurrence-free survival; OS, overall survival; PFS, progression failure-free survival; RRFS, regional recurrence-free survival.

$87.5 \%$, respectively (Figure 1). The overall 3-year OS rates for the patients were $100 \%$ for stage T1-2 and $79.6 \%$ for stage T3-4 $(P=0.039)$, respectively (Figure 2 ). The overall 3-year OS rates for the patients with adjuvant chemotherapy were $95.8 \%$ vs $55.6 \%$ for patients without adjuvant chemotherapy (Figure 3).

Altogether seven patients had experienced treatment failure. There were two patients with local recurrence alone and both of them died of uncontrolled epistaxis after re-irradiation. One patient had regional recurrence alone and received salvage surgery. He was still clinically free of disease at the last assessment. Three patients developed one or more distant metastases and all of them died of disease progression. One patient had both regional and distant failure who was still alive but failed to have a remission after aggressive chemotherapy. Details are shown in Table 2.

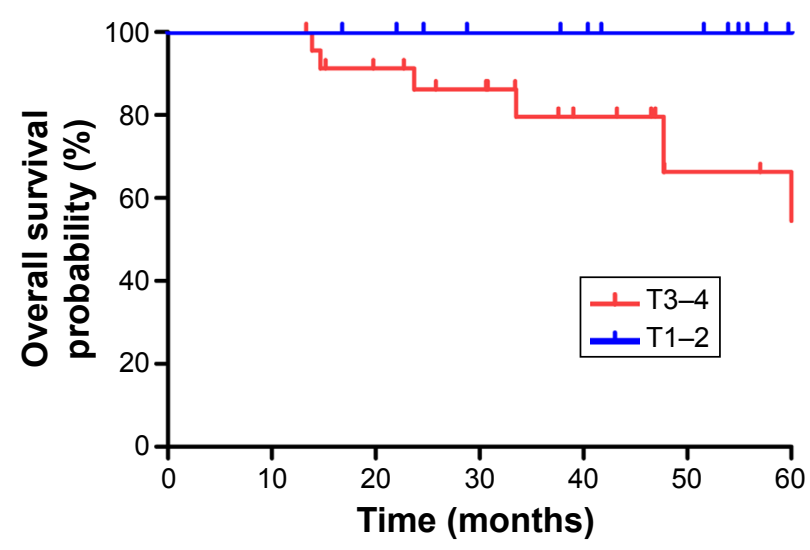

Figure 2 Kaplan-Meier curves showing overall survival for patients with TI-2 and T3-4 stage.

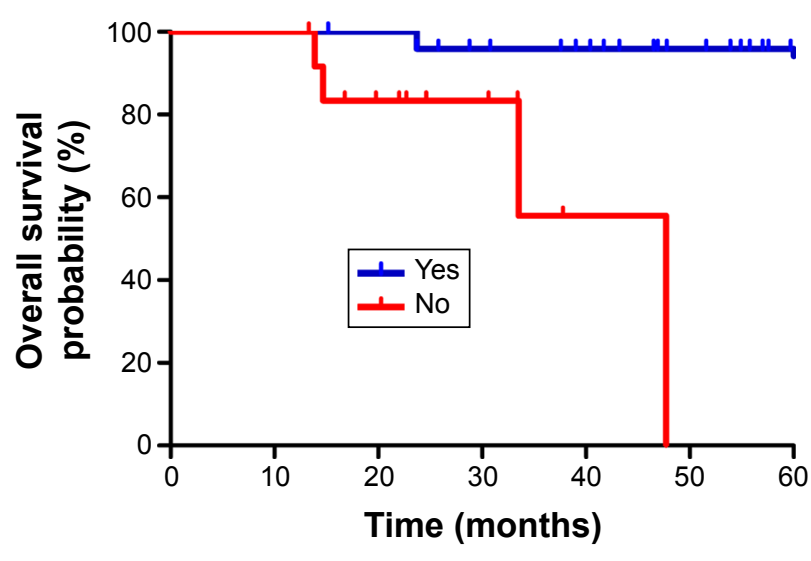

Figure 3 Kaplan-Meier curves showing OS for patients with adjuvant chemotherapy and without adjuvant chemotherapy.

A total of six deaths were recorded at the last follow-up, other than the five mentioned, the remaining one died of epilepsy attack, a cause not cancer-related and with no evidence of disease progression.

\section{Prognostic factors}

Potential prognostic factors including age, sex, stage, adjusted tumor $(\mathrm{T})$ stage and lymph node $(\mathrm{N})$ stage, chemotherapy, cycles of nimotuzumab as well as response at 3 months after the completion of RT were evaluated. Univariate analysis showed that $\mathrm{T}$ stage and adjuvant chemotherapy were significant prognostic factors for OS, favoring those with T1-2 disease and addition of adjuvant chemotherapy. However, patients with advanced T stage (T3-4) showed a marginally negative trend on OS vs those with earlier $\mathrm{T}$ stage in multivariate analysis (Table 3). We attributed that to the relatively small sample size and short time follow-up.

\section{Toxicity}

The most common radiation-related acute adverse effects were stomatitis and skin reaction (Table 4). Oral mucositis was reported as grade 3 in severity in 14 (36.8\%) patients. No grade 3 dermatitis was observed within the RT field. The majority of toxicities were mild or moderate, and patients

Table 2 Site and incidence of treatment failure

\begin{tabular}{ll}
\hline Site & $\begin{array}{l}\text { Number of patients } \\
(\mathbf{n}=\mathbf{7})\end{array}$ \\
\hline Local only & 2 \\
Regional only & $\mathrm{I}$ \\
Regional and distant & $\mathrm{I}$ \\
Distant only & \\
$\quad$ Lung, bone, brain & $\mathrm{I}$ \\
Bone only & $\mathrm{I}$ \\
\hline
\end{tabular}

Note: aBrain metastases were histologically proven. 
Table 3 Impact of prognostic factors on treatment results by univariate analysis

\begin{tabular}{|c|c|c|c|c|c|c|c|c|c|c|}
\hline \multirow[t]{2}{*}{$\overline{\text { Variable }}$} & \multicolumn{2}{|c|}{ 3-year LRFS } & \multicolumn{2}{|c|}{ 3-year RRFS } & \multicolumn{2}{|c|}{ 3-year DMFS } & \multicolumn{2}{|c|}{ 3-year PFS } & \multicolumn{2}{|c|}{ 3-year OS } \\
\hline & $\%$ & $P$-value & $\%$ & $P$-value & $\%$ & $P$-value & $\%$ & $P$-value & $\%$ & $P$-value \\
\hline \multicolumn{11}{|l|}{ Sex } \\
\hline Male & 95.2 & 0.742 & 88.9 & 0.279 & 84.0 & 0.136 & 69.6 & 0.130 & 80.6 & 0.313 \\
\hline Female & 88.9 & & 100 & & 100 & & 88.9 & & 100 & \\
\hline \multicolumn{11}{|l|}{ Age } \\
\hline$<46$ & 93.8 & 0.893 & 100 & 0.181 & 83.3 & 0.254 & 77.4 & 0.780 & 80.1 & 0.590 \\
\hline$\geq 46$ & 92.3 & & 86.9 & & 95.0 & & 75.9 & & 94.7 & \\
\hline \multicolumn{11}{|l|}{ Stage } \\
\hline III & 100 & 0.154 & 85.9 & 0.157 & 90.0 & 0.957 & 81.8 & 0.453 & 93.8 & 0.157 \\
\hline IV & 85.9 & & 100 & & 88.9 & & 70.7 & & 81.5 & \\
\hline \multicolumn{11}{|l|}{ T stage } \\
\hline TI-2 & 100 & 0.226 & 100 & 0.243 & 100 & 0.114 & 100 & 0.016 & 100 & 0.039 \\
\hline T3-4 & 87.7 & & 88.2 & & 83.3 & & 61.9 & & 79.6 & \\
\hline \multicolumn{11}{|l|}{$\mathrm{N}$ stage } \\
\hline No-I & 100 & 0.493 & 75.0 & 0.261 & 100 & 0.289 & 65.6 & 0.774 & 87.5 & 0.852 \\
\hline N2-3 & 91.2 & & 96.2 & & 86.7 & & 78.4 & & 87.9 & \\
\hline \multicolumn{11}{|l|}{ Adj CHT } \\
\hline Yes & 100 & 0.005 & 91.0 & 0.449 & 88.0 & 0.677 & 83.4 & 0.186 & 95.8 & 0.001 \\
\hline No & 60.0 & & 100 & & 92.3 & & 50.3 & & 55.6 & \\
\hline \multicolumn{11}{|l|}{ Nimotuzumab } \\
\hline$<6$ cycles & 80.0 & 0.191 & 100 & 0.507 & 87.5 & 0.853 & 70.0 & 0.697 & 68.6 & 0.291 \\
\hline$\geq 6$ cycles & 94.7 & & 91.4 & & 90.0 & & 77.0 & & 92.8 & \\
\hline
\end{tabular}

Abbreviations: Adj CHT, adjuvant chemotherapy; cycles, cycles of nimotuzumab; DMFS, distant metastasis-free survival; LRFS, local recurrence-free survival; OS, overall survival; PFS, progression failure-free survival; RRFS, regional recurrence-free survival.

recovered rapidly with or without symptomatic medication. During concurrent phase, no acneiform eruptions were found among these subjects.

Xerostomia was the most common late effect, and the degree of xerostomia appeared to decrease with time. At the time of analysis, most patients developed mild-to-moderate xerostomia and four patients had no subjective complaints of dry mouth.

Seven patients suffered unilateral or bilateral hearing impairment. One patient developed temporal lobe necrosis, diagnosed by MRI examination in the follow-up.

\section{Discussion}

This study showed that nimotuzumab could be safely combined with IMRT in the curative treatment of LA NPC. This approach was associated with a relatively low rate of RT-related acute skin and mucosal toxic effects. In our study,

Table 4 Frequency of grade 2, 3, or 4 AEs during radiotherapy

\begin{tabular}{llll}
\hline AEs & $\begin{array}{l}\text { Grade 2 } \\
\text { n (\%) }\end{array}$ & $\begin{array}{l}\text { Grade 3 } \\
\text { n (\%) }\end{array}$ & $\begin{array}{l}\text { Grade 4 } \\
\text { n (\%) }\end{array}$ \\
\hline Stomatitis & $20(52.6)$ & $14(36.8)$ & $0(0.0)$ \\
Dermatitis & $4(10.5)$ & $0(0.0)$ & $0(0.0)$ \\
Vomit & I (2.6) & $2(5.3)$ & $0(0.0)$ \\
\hline
\end{tabular}

Abbreviation: AEs, adverse events. grade 3 radiation-induced mucositis accounted for $36.8 \%$ of treated people and no grade 4 mucositis was observed. No skin rash and infusion reaction were observed. The objective response rate at 3 months after the completion of radiation was $100 \%$, with a 3 year OS of $87.5 \%$. The tumor response rate and preliminary survival data of this combined strategy compared favorably with those previously reported by other centers. ${ }^{19-21}$

For many years, CRT is the standard treatment for LA NPC. The combination of radiation and concurrent chemotherapy has been demonstrated to be superior to radiation alone for LA NPC in both local control and OS. Table 5 summarizes the key features and results achieved at various centers. ${ }^{19-21}$ The 3-year OS reported previously ranged from $83 \%$ to $94 \%$, which were quite promising outcomes. But the CRT series had significantly exacerbated RT-related grade 3 or worse mucositis, with an incidence of $41 \%-78 \% .{ }^{21-23}$ Therefore, in this study, we evaluated the safety and efficacy of nimotuzumab as a concurrent agent to IMRT, aiming to find clinical merits especially in mitigating toxicities.

Given to the high rate of EGFR overexpression as well as its cognate and the consequent negative influences in treatment responses and survival in HNSCC, the EGFR antagonists have shown encouraging clinical outcomes in palliative and curative settings. A previous work showed 
Table 5 Treatment outcomes and adverse effects in selected studies treated with IMRT

\begin{tabular}{|c|c|c|c|c|}
\hline & \multicolumn{4}{|l|}{ Center } \\
\hline & $\mathrm{NCC}^{19}$ & $\mathrm{MKSCC}^{20}$ & $\mathbf{P W H}^{21}$ & Our series \\
\hline Number of patients & 195 & 74 & 63 & 38 \\
\hline Year & 2009 & 2006 & 2004 & 2015 \\
\hline Median follow-up (months) & 36.5 & 35 & 29 & 39.7 \\
\hline Stage III/IV (\%) & 63 & 77 & 57 & 100 \\
\hline Con CHT (\%) & 57 & 93 & 25 & None \\
\hline Adj CHT (\%) & 35 & 93 & 0 & 65.8 \\
\hline Dose to GTV-P (Gy) & 70 & 70 & 66 & $66 / 70.4$ \\
\hline Dose per fraction (Gy) & $2.0-2.12$ & 2.34 & 2 & 2.2 \\
\hline Fraction & $33-35$ & 30 & 33 & $30 / 32$ \\
\hline Add boosts & $\mathrm{Br} 10 \%$ & CB $80 \%$ & $\mathrm{Br} / 3 \mathrm{D} 56 \%$ & None \\
\hline Time point (years) & 3 & 3 & 3 & 3 \\
\hline LRFS (\%) & 90 & 91 & 92 & 92.8 \\
\hline RRFS (\%) & $N R$ & 93 & 98 & 92.9 \\
\hline DMFS (\%) & 89.2 & 78 & 79 & 89.5 \\
\hline PFS (\%) & 79 & 67 & NR & 76.3 \\
\hline OS (\%) & 94.3 & 83 & 90 & 87.5 \\
\hline Grade $3 / 4$ mucositis (\%) & 29 & NR & 41 & 36.8 \\
\hline Grade $3 / 4$ dermatitis (\%) & 2 & NR & 7 & 0 \\
\hline
\end{tabular}

Abbreviations: Adj CHT, adjuvant chemotherapy; Br, brachytherapy boost; CB, concomitant boost; Con CHT, concurrent chemotherapy; DMFS, distant metastasis-free survival; GTV-P, gross primary tumor volume of the nasopharynx; LRFS, local recurrence-free survival; MSKCC, Memorial Sloan Kettering Cancer Center; NCC, National Cancer Center; NR, not reported; OS, overall survival; PFS, progression failure-free survival; PWH, Prince of Wales Hospital; RRFS, regional recurrence-free survival.

that the rate of EGFR overexpression in NPC could be as high as $94 \% .{ }^{8}$ Cetuximab, as the most commonly used monoclonal antibody, has been approved for the treatment of HNSCC in combination with radiation for locoregionally advanced disease and in combination with cisplatin-based chemotherapy for the first-line treatment of recurrent/ metastatic disease. ${ }^{10}$ Bonner's study demonstrated that cetuximab significantly improved locoregional tumor control and survival when concomitantly used with RT compared with RT alone. ${ }^{24}$ Other cetuximab-based studies in NPC also showed promising clinic outcomes, with 2-year PFS of $86.5 \%-89.3 \%$ and 3 -year OS of $90.9 \%{ }^{11,13,25}$ However, all the studies reported unexpectedly higher incidence of grade 3-4 oral mucositis (56\%-87\%) and RT-related dermatitis $(20 \%-25 \%) .{ }^{11-13,25}$ The frequency of oral mucositis was twice as common as the data in the current study. Grade 3 RT-related dermatitis (33\%) was much more common than that in our study. Furthermore, administration of cetuximab resulted in more than $90 \%$ of treated patients being asymptomatic or having an itchy acneiform rash. ${ }^{11-13,25}$ In contrast to that, no patients suffered severe skin rash during the treatment. To minimize cetuximab-related toxicities, novel EGFR-targeted agent was warranted.

Nimotuzumab, as a humanized Immunoglobulin G1 (IgG1) isotype monoclonal antibody with unique safety profile and low skin toxicity, has shown clinical benefits in the management of non-NPC HNSCC. The advantage of the drug is that the affinity constant is quite lower than that of cetuximab, allowing for high tumor uptake and low normal tissues uptake. ${ }^{26}$ Nimotuzumab requires bivalent binding for stable attachment, which renders the agent to selectively binding to tumors with moderate-to-high EGFR levels. When EGFR expression is low as on the normal tissues, cetuximab still had high ability of binding because of its higher affinity constant. ${ }^{26}$ In a phase II study of nimotuzumab plus RT for stage III-IV unresectable HNSCC, the nimotuzumab add-on group was superior to the placebo add-on group, resulting in a significantly higher complete remission rate ( $59.5 \%$ vs $34.2 \%$, respectively) and longer median survival (20.31 vs 11.77 months, respectively) without exacerbation of radiation-related adverse events. ${ }^{15}$ Similar results were also observed in another phase II study where nimotuzumab was combined with RT alone or CRT in treatment-naïve LA HNSCC. ${ }^{16}$ The phase II study was conducted in 2014 as a randomized, open-labeled, four-armed, multicenter investigation that employed a cohort of 92 LA HNSCC. The patients were divided into two arms, with half of them receiving CRT and the other half $\mathrm{RT}$ alone. In each arm, the participants were randomized to receive treatment regimen with or without weekly nimotuzumab in addition to their scheduled RT or CRT. The objective response rate (the sum of CR and partial remission [PR]) was significantly higher when nimotuzumab was added in both CRT arm (100\% vs $70 \%, P=0.020)$ and RT arm $(76.4 \%$ vs $37 \%, P=0.023)$. 
Also, the addition of nimotuzumab in the CRT arm gave an improvement of 5 -year OS rate by $31 \%$ (57\% vs $26 \%$, $P=0.03)$ and lowered risk of death by $64 \%$ (HR $=0.36,95 \%$ CI: $0.37,1.56)$. In the RT arm, a trend of improvement in OS rate at 5 years was observed in patients with nimotuzumab (39\% vs $26 \%, P>0.5$ ), albeit not reaching statistical significance. In the former study, the incidence of overall oral mucositis and dermatitis in the nimotuzumab group were only $20.1 \%$ and $10.3 \%$, respectively. We attributed the low incidence to the relatively low prescribed radiation dose (with a mean cumulative dose of 6,030 cGy). In the latter study, there were no significant increases in the incidence of severe mucositis and dermatitis in the four arms and skin rash was observed in only $6.5 \%$ of the patients. All of these indicated that nimotuzumab plus RT could be selected in the design of the clinical trial of NPC.

Our experience suggested that nimotuzumab might be a good alternative for locoregionally advanced outpatients or patients who could not tolerate CRT. However, our results should be regarded as preliminary owing to the relatively small sample size and bias in patient selection.

\section{Conclusion}

In conclusion, our study showed that concurrent administration of nimotuzumab with RT was well-tolerated and showed encouraging clinical activities. Further randomized, controlled, multicenter phase III clinical trials are needed to confirm the ultimate therapeutic gain.

\section{Future perspective}

More clinical data of randomized trials directly comparing IMRT plus nimotuzumab with IMRT plus concurrent chemotherapy are needed to confirm the clinical efficacy of nimotuzumab.

\section{Disclosure}

The authors report no conflicts of interest in this work.

\section{References}

1. Al-Sarraf M, LeBlanc M, Giri PG, et al. Chemoradiotherapy versus radiotherapy in patients with advanced nasopharyngeal cancer: phase III randomized intergroup study 0099. J Clin Oncol. 1998;16(4): 1310-1317.

2. Lee AW, Lau WH, Tung SY, et al. Preliminary results of a randomized study on therapeutic gain by concurrent chemotherapy for regionallyadvanced nasopharyngeal carcinoma: NPC-9901 Trial by the Hong Kong Nasopharyngeal Cancer Study Group. J Clin Oncol. 2005;23(28): 6966-6975.

3. Lin JC, Jan JS, Hsu CY, Liang WM, Jiang RS, Wang WY. Phase III study of concurrent chemoradiotherapy versus radiotherapy alone for advanced nasopharyngeal carcinoma: positive effect on overall and progression-free survival. J Clin Oncol. 2003;21(4):631-637.
4. Wee J, Tan EH, Tai BC, et al. Randomized trial of radiotherapy versus concurrent chemoradiotherapy followed by adjuvant chemotherapy in patients with American Joint Committee on Cancer/International Union against cancer stage III and IV nasopharyngeal cancer of the endemic variety. J Clin Oncol. 2005;23(27):6730-6738.

5. Lee N, Xia P, Quivey JM, et al. Intensity-modulated radiotherapy in the treatment of nasopharyngeal carcinoma: an update of the UCSF experience. Int J Radiat Oncol Biol Phys. 2002;53(1):12-22.

6. Herbst RS, Shin DM. Monoclonal antibodies to target epidermal growth factor receptor-positive tumors: a new paradigm for cancer therapy. Cancer. 2002;94(5):1593-1611.

7. Chua DT, Nicholls JM, Sham JS, Au GK. Prognostic value of epidermal growth factor receptor expression in patients with advanced stage nasopharyngeal carcinoma treated with induction chemotherapy and radiotherapy. Int J Radiat Oncol Biol Phys. 2004;59(1):11-20.

8. Ma BB, Poon TC, To KF, et al. Prognostic significance of tumor angiogenesis, Ki 67, p53 oncoprotein, epidermal growth factor receptor and HER2 receptor protein expression in undifferentiated nasopharyngeal carcinoma - a prospective study. Head Neck. 2003;25(10): 864-872.

9. Leong JL, Loh KS, Putti TC, Goh BC, Tan LK. Epidermal growth factor receptor in undifferentiated carcinoma of the nasopharynx. Laryngoscope. 2004;114(1):153-157.

10. Bonner JA, Harari PM, Giralt J, et al. Radiotherapy plus cetuximab for locoregionally advanced head and neck cancer: 5-year survival data from a phase 3 randomised trial, and relation between cetuximabinduced rash and survival. Lancet Oncol. 2010;11(1):21-28.

11. Ma BB, Kam MK, Leung SF, et al. A phase II study of concurrent cetuximab-cisplatin and intensity-modulated radiotherapy in locoregionally advanced nasopharyngeal carcinoma. Ann Oncol. 2012;23(5): 1287-1292.

12. He X, Xu J, Guo W, Jiang X, Wang X, Zong D. Cetuximab in combination with chemoradiation after induction chemotherapy of locoregionally advanced nasopharyngeal carcinoma: preliminary results. Future Oncol. 2013;9(10):1459-1467.

13. Feng HX, Guo SP, Li GR, et al. Toxicity of concurrent chemoradiotherapy with cetuximab for locoregionally advanced nasopharyngeal carcinoma. Med Oncol. 2014;31(9):170.

14. Crombet T, Osorio M, Cruz T, et al. Use of the humanized anti-epidermal growth factor receptor monoclonal antibody h-R3 in combination with radiotherapy in the treatment of locally advanced head and neck cancer patients. J Clin Oncol. 2004;22(9):1646-1654.

15. Rodriguez MO, Rivero TC, Del CBR, et al. Nimotuzumab plus radiotherapy for unresectable squamous-cell carcinoma of the head and neck. Cancer Biol Ther. 2010;9(5):343-349.

16. Reddy BK, Lokesh V, Vidyasagar MS, et al. Nimotuzumab provides survival benefit to patients with inoperable advanced squamous cell carcinoma of the head and neck: a randomized, open-label, phase IIb, 5-year study in Indian patients. Oral Oncol. 2014;50(5):498-505.

17. Nowak PJ, Wijers OB, Lagerwaard FJ, Levendag PC. A threedimensional CT-based target definition for elective irradiation of the neck. Int J Radiat Oncol Biol Phys. 1999;45(1):33-39.

18. Gregoire V, Levendag P, Ang KK, et al. CT-based delineation of lymph node levels and related CTVs in the node-negative neck: DAHANCA, EORTC, GORTEC, NCIC, RTOG consensus guidelines. Radiother Oncol. 2003;69(3):227-236.

19. Tham IW, Hee SW, Yeo RM, et al. Treatment of nasopharyngeal carcinoma using intensity-modulated radiotherapy - The National Cancer Centre Singapore experience. Int J Radiat Oncol Biol Phys. 2009;75(5): 1481-1486.

20. Wolden SL, Chen WC, Pfister DG, Kraus DH, Berry SL, Zelefsky MJ. Intensity-modulated radiation therapy (IMRT) for nasopharynx cancer: update of the Memorial Sloan-Kettering experience. Int J Radiat Oncol Biol Phys. 2006;64(1):57-62.

21. Kam MK, Teo PM, Chau RM, et al. Treatment of nasopharyngeal carcinoma with intensity-modulated radiotherapy: the Hong Kong experience. Int J Radiat Oncol Biol Phys. 2004;60(5):1440-1450. 
22. Kwong DL, Sham JS, Leung LH, et al. Preliminary results of radiation dose escalation for locally advanced nasopharyngeal carcinoma. Int J Radiat Oncol Biol Phys. 2006;64(2):374-381.

23. Ng WT, Lee MC, Hung WM, et al. Clinical outcomes and patterns of failure after intensity-modulated radiotherapy for nasopharyngeal carcinoma. Int J Radiat Oncol Biol Phys. 2011;79(2):420-428.

24. Bonner JA, Harari PM, Giralt J, et al. Radiotherapy plus cetuximab for squamous-cell carcinoma of the head and neck. $N$ Engl J Med. 2006; 354(6):567-578.
25. Niu X, Hu C, Kong L. Experience with combination of cetuximab plus intensity-modulated radiotherapy with or without chemotherapy for locoregionally advanced nasopharyngeal carcinoma. J Cancer Res Clin Oncol. 2013;139(6):1063-1071.

26. Ramakrishnan MS, Eswaraiah A, Crombet T, et al. Nimotuzumab, a promising therapeutic monoclonal for treatment of tumors of epithelial origin. mAbs. 2009;1(1):41-48.

\section{Publish your work in this journal}

OncoTargets and Therapy is an international, peer-reviewed, open access journal focusing on the pathological basis of all cancers, potential targets for therapy and treatment protocols employed to improve the management of cancer patients. The journal also focuses on the impact of management programs and new therapeutic agents and protocols on

\section{Dovepress}

patient perspectives such as quality of life, adherence and satisfaction. The manuscript management system is completely online and includes a very quick and fair peer-review system, which is all easy to use. Visit http://www.dovepress.com/testimonials.php to read real quotes from published authors.

\footnotetext{
Submit your manuscript here: http://www.dovepress.com/oncotargets-and-therapy-journal
} 